\title{
Pseudospectral method for the analysis of diffractive optical elements
}

\author{
P. G. Dinesen \\ Department of Optics and Fluid Dynamics, Risø National Laboratory, OFD-128, P.O. Box 49, \\ DK-4000 Roskilde, Denmark \\ J. S. Hesthaven \\ Division of Applied Mathematics, Brown University, Box F, Providence, Rhode Island 02912 \\ J. P. Lynov and L. Lading \\ Department of Optics and Fluid Dynamics, Risø National Laboratory, OFD-128, P.O. Box 49, \\ DK-4000 Roskilde, Denmark
}

\begin{abstract}
Received August 4, 1998; revised manuscript received November 30, 1998; accepted December 2, 1998
\end{abstract}
\begin{abstract}
A pseudospectral method for the analysis of diffractive optical elements is presented. This method is a fullvectorial direct solution of the time-domain Maxwell equations based on a spectral approximation of the spatial derivatives employed within a multidomain framework. The method exhibits little numerical dispersion, and only a few points per wavelength are needed to accurately resolve the propagation of the optical field over long distances. A comparison with the analytic solution for a thin-film waveguide is performed, and examples of analyses of grating couplers are given to demonstrate the feasibility of the method. (C) 1999 Optical Society of America [S0740-3232(99)00705-X]

OCIS codes: $050.1960,000.4430$.
\end{abstract}

\section{INTRODUCTION}

The design of diffractive optical elements (DOE's) has attracted an increasing amount of interest over the past three decades, and both scalar and full-vectorial methods for modeling wave propagation in such elements have been developed. The successful use of scalar diffraction theory requires that the feature size of the DOE be much larger than the optical wavelength, and, given the emerging interest in resonant structures thanks to their superior properties in terms of diffraction efficiencies, ${ }^{1}$ using scalar diffraction theory is clearly no longer sufficient. Thus, for the analysis of resonant structures, methods for the rigorous solution of Maxwell's equations have been developed. Up until now, the majority of the work has been limited to the analysis of purely periodic structures of infinite extent by techniques such as rigorous coupledwave analysis. ${ }^{2}$ As for finite aperiodic structures, a number of methods using both integral equation and differential equation methods have recently been presented. ${ }^{3-7}$ While integral methods must be considered inappropriate for the analysis of grating couplers because of the multiple dielectric layers in such structures, the finitedifference time-domain method (FDTD) ${ }^{7}$ is an alternative. This method has, however, a serious drawback that will be discussed below.

As for the analysis of focusing grating couplers (FGC's), geometrical-optical methods such as those of Refs. 8 and 9 have been used. A more rigorous analysis using eigen- mode expansions was presented in Ref. 10, and the use of the FDTD was extensively described in a recent paper. ${ }^{11}$

In this study we present what we believe is the first application of pseudospectral methods to the analysis of DOE's and, more specifically, of grating couplers. Spectral methods have been developed and refined within the field of fluid mechanics for many years, but it was not until recently that this method was introduced to solving problems within electromagnetics. In Ref. 12 the pseudospectral scheme was applied to the modeling of electromagnetic scattering.

Our pseudospectral scheme computes a direct solution of the Maxwell equations in the time domain. The key element in the method is that the spatial derivatives of the field components are estimated by use of a spectral scheme by which information from many, typically 16-20, points are used to calculate the derivative, which as we shall demonstrate ensures a high accuracy. In the FDTD one approximates the spatial derivatives by using only information from two neighbor points, and it is well known that a serious drawback of the FDTD is the inherent numerical dispersion. ${ }^{13}$ This numerical dispersion leads to the accumulation of phase errors as the field propagates. While the FDTD may therefore provide accurate results by use of a reasonable resolution for small problems, as the size of the problem increases, an increasing resolution must also be used to suppress the global phase error. For grating couplers typically extending over several hun- 
dreds of wavelengths in the direction of propagation of the guided wave, the interplay between the radiated field and the guided wave is critically dependent on the phase behavior, and hence the applicability of the FDTD is therefore limited for such large problems. Our pseudospectral approach, by contrast, shows no numerical dispersion, and therefore it is not necessary to increase the resolution as the size of the computational domain is increased. As we shall demonstrate, our scheme can accurately model the field propagation with as little as 7-10 points/ wavelength.

Another drawback of the FDTD is that interfaces are traditionally approximated through a staircase approximation that gives rise to significant errors and spurious fields. In our pseudospectral approach, we use a curvilinear coordinate mapping so that modulated interfaces can be accurately resolved.

As a final advantage of our scheme, we note that it is well suited to parallel implementation owing to the use of a domain decomposition scheme. ${ }^{14}$

An example of an integrated optical component that uses focusing grating couplers is the integrated optical time of flight velocimeter, shown in Fig. 1. This device comprises two FGC's for focused in-plane and out-of-plane coupling of light between a semiconductor laser-detector and free space.

In Section 2 we briefly outline the equations that we wish to solve, and in Section 3 our pseudospectral approach is described. Section 4 is devoted to numerical results for test cases, with examples including a FGC, while the conclusions can be found in Section 5 .

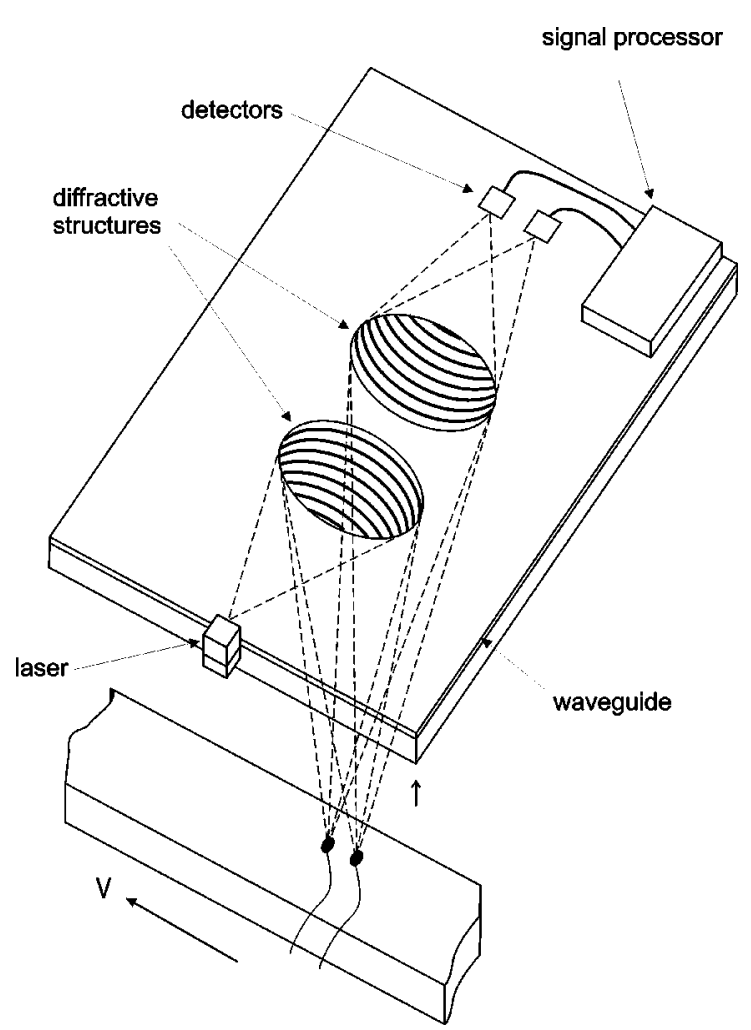

Fig. 1. Time-of-flight velocimeter comprising two FGC's.

\section{PRELIMINARIES}

We shall in the present study restrict ourselves to the problem of solving Maxwell's equations in two dimensions, and, furthermore, we shall limit the analysis to the transverse-electric case. Hence, in source-free dielectric layers, the equations become

$$
\begin{aligned}
\frac{\partial \tilde{H}_{z}}{\partial \tilde{t}} & =-\frac{c}{Z_{0}} \frac{\partial \tilde{E}_{y}}{\partial \tilde{x}}, \\
\frac{\partial \tilde{H}_{x}}{\partial \tilde{t}} & =\frac{c}{Z_{0}} \frac{\partial \tilde{E}_{y}}{\partial \tilde{z}}, \\
\frac{\partial \tilde{E}_{y}}{\partial \tilde{t}} & =c Z_{0} \frac{1}{n^{2}}\left(\frac{\partial \tilde{H}_{x}}{\partial \tilde{z}}-\frac{\partial \tilde{H}_{z}}{\partial \tilde{x}}\right),
\end{aligned}
$$

where $\tilde{H}_{z}$ and $\tilde{H}_{x}$ represent the dimensional magnetic fields in the plane, while $\widetilde{E}_{y}$ refers to the perpendicular component of the electric field. We have also introduced the free-space impedance, $Z_{0}$; the vacuum speed of light, $c$; and the index of refraction $n(z, x)$.

We are interested only in steady-state solutions of monochromatically driven problems, and therefore it may seem undesirable to compute the time-domain solution. However, the hyperbolic nature of the time-domain Maxwell equations facilitates a multidomain formulation, as we shall discuss below.

Since we use a monochromatic incident field, frequency $\nu$, we may conveniently introduce the normalized variables

$$
t=\tilde{t} \nu, \quad x=\tilde{x} / \lambda, \quad z=\tilde{z} / \lambda .
$$

By also introducing the normalized field components

$$
H_{x}=\tilde{H}_{x}, \quad H_{y}=\tilde{H}_{y}, \quad E_{z}=Z_{0}{ }^{-1} \widetilde{E}_{z},
$$

we arrive at the nondimensional transverse-electric equations

$$
\begin{aligned}
\frac{\partial H_{z}}{\partial t} & =-\frac{\partial E_{y}}{\partial x}, \\
\frac{\partial H_{x}}{\partial t} & =\frac{\partial E_{y}}{\partial z}, \\
\frac{\partial E_{y}}{\partial t} & =\frac{1}{n^{2}}\left(\frac{\partial H_{x}}{\partial z}-\frac{\partial H_{z}}{\partial x}\right),
\end{aligned}
$$

The $\lambda$ introduced above is the free-space wavelength, $\lambda$ $=c / \nu$.

We consider the materials involved to be nonmagnetic and lossless such that the boundary conditions between layers of different dielectric constants become

$$
E_{y}{ }^{1}=E_{y}{ }^{2}, \quad \hat{\mathbf{n}} \times \mathbf{H}^{1}=\hat{\mathbf{n}} \times \mathbf{H}^{2}, \quad \hat{\mathbf{n}} \cdot \mathbf{H}^{1}=\hat{\mathbf{n}} \cdot \mathbf{H}^{2},
$$

where the superscripts refer to the field components in two neighboring layers, while $\hat{\mathbf{n}}$ signifies the unit vector normal to the interface. 


\section{NUMERICAL SCHEME}

The pseudospectral method that we propose is based on the following key elements: estimation of spatial derivatives by a Chebyshev collocation scheme, adaptation to irregular geometries by a curvilinear multidomain formulation, patching of local solutions by use of characteristic variables, and time integration of the solution. Each of these steps is described in this section.

The time integration is accomplished by a five-stage fourth-order low-storage Runge-Kutta scheme that was developed in Ref. 15. Although it requires an extra stage for the completion of the step as compared with the standard fourth-order Runge-Kutta scheme, it has a slightly larger stability region, implying that the total work is kept approximately constant. However, only one storage level is required for implementing the scheme.

\section{A. Chebyshev Collocation Method}

The Chebyshev polynomial of order $k$ is defined as

$$
T_{k}(z)=\cos \left(k \cos ^{-1} z\right),
$$

where $|z| \leqslant 1$. We shall consider collocation methods in which the $N+1$ collocation points are chosen to be the Chebyshev-Gauss-Lobatto points that appear as the roots of the polynomial $\left(1-z^{2}\right) T_{N}{ }^{\prime}(z)$, i.e.,

$$
z_{i}=-\cos \left(\frac{i \pi}{N}\right), \quad 0 \leqslant i \leqslant N .
$$

When applying a Chebyshev collocation method, one approximates a function, $f(z)$, by a grid function, $f_{i}$ $=f\left(z_{i}\right)$, where the grid points are the Gauss-Lobatto points $z_{i}$. We construct a global $N$ th-order Chebyshev interpolant, $I_{N}$, to obtain the approximation of the function

$$
\left(I_{N} f\right)(z)=\sum_{i=0}^{N} f_{i} g_{i}(z)
$$

The interpolating Chebyshev-Lagrange polynomials are given as

$$
g_{i}(z)=\frac{\left(1-z^{2}\right) T_{N}{ }^{\prime}(z)(-1)^{i+1}}{c_{i} N^{2}\left(z-z_{i}\right)},
$$

where $c_{0}=c_{N}=2$ and $c_{i}=1$ for $1 \leqslant i \leqslant N-1$.

To seek approximate solutions, $\left(I_{N} f\right)(z)$, to a partial differential equation, we ask that the equation be satisfied in a collocation sense, i.e., at the collocation points. Hence we need to obtain values of the spatial derivatives at the collocation points. This is accomplished by approximation of the continuous differential operator by a matrix operator with the entries given as

$$
D_{i j}=g_{j}{ }^{\prime}\left(z_{i}\right)
$$

such that the derivative of $f$ at a collocation point, $z_{i}$, is approximated as

$$
\frac{\mathrm{d} f}{\mathrm{~d} z}\left(z_{i}\right) \approx \frac{\mathrm{d}\left(I_{N} f\right)}{\mathrm{d} z}\left(z_{i}\right)=\sum_{j=0}^{N} D_{i j} f\left(z_{j}\right),
$$

and likewise for higher derivatives. For the explicit expressions of the entries of the matrix operator and for further details on collocation methods, we refer the reader to Ref. 16.

To extend the outlined scheme to two dimensions we construct a two-dimensional approximation by a tensor product,

$$
\left(I_{N, M} f\right)(z, x)=\sum_{i=0}^{N} \sum_{j=0}^{M} f\left(z_{i}, x_{j}\right) g_{i}(z) g_{j}(x),
$$

where a grid, $x_{i}$, along $x$ is introduced. By this approach derivatives are computed through the use of onedimensional differentiation matrices and matrix-matrix products. A tensor-product formulation requires that $f$ be given on a rectangular grid, a restriction that we overcome by introducing a curvilinear representation.

\section{B. Curvilinear Representation}

We assume the existence of a smooth nonsingular mapping function, $\Psi$, relating the $(z, x)$ coordinate system to the general curvilinear coordinate $\operatorname{system}(\xi, \eta)$ such that

$$
\xi=\xi(z, x), \quad \eta=\eta(z, x),
$$

as illustrated in Fig. 2.

In the curvilinear representation, Eqs. (4) can be written as

$$
\frac{\partial \mathbf{q}}{\partial t}+A(\nabla \xi) \frac{\partial \mathbf{q}}{\partial \xi}+A(\nabla \eta) \frac{\partial \mathbf{q}}{\partial \eta}=0,
$$

where $\mathbf{q}=\left(H_{z}, H_{x}, E_{y}\right)^{T}$ is the state vector. The general operator, $(\mathbf{n})$, with $\mathbf{n}=\left(n_{z}, n_{x}\right)$ representing the local metric, is given as

$$
A(n)=\left(\begin{array}{ccc}
0 & 0 & n_{x} \\
0 & 0 & -n_{z} \\
n_{x} n^{-2} & -n_{z} n^{-2} & 0
\end{array}\right),
$$

where we recall that $n$ refers to the local index of refraction. We shall return to operator $A$ and its significance shortly.

To establish a one-to-one correspondence between the unit square on which we perform the calculation of spatial derivatives and the general quadrilateral, we construct the local map by using transfinite blending functions. ${ }^{17}$ We refer the reader to Ref. 18 for a thorough treatment of this procedure within the present context. Once the map $\Psi$ is constructed, we can compute the metric of the mapping and outward point normal vectors at all the grid points on the edges of the quadrilateral.

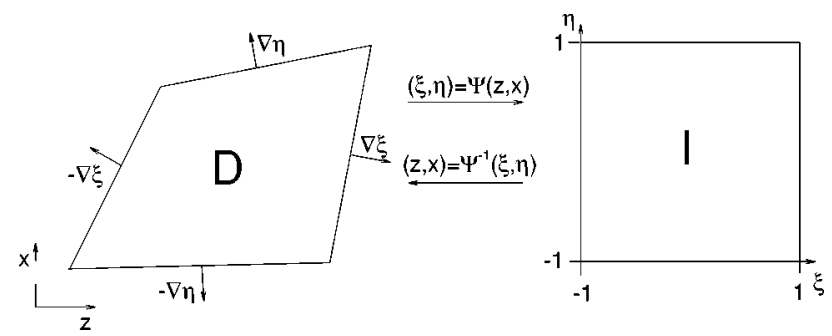

Fig. 2. Mapping, $\Psi_{1}$, between physical coordinates $(z, x)$ and general curvilinear coordinates $(\xi, \eta)$. 


\section{Multidomain Formulation}

We wish to solve Eq. (14) within a general computational domain, $\Omega \in R^{2}$, in the $(z, x)$ plane. We construct $\Omega$ by using $K$ nonoverlapping general curvilinear quadrilaterals. In each of these quadrilaterals we may then compute the spatial derivatives and advance the system one time step, as discussed above.

In constructing the global solution from all the local solutions, we encounter two different types of interface requiring different techniques of patching. On boundaries between domains of different refractive indices, we use the physical boundary conditions of Eqs. (5), which are enforced strongly. On boundaries between subdomains of the same material, we take advantage of the hyperbolic nature of Eq. (14) in constructing the global solution. The operator $A$ in this equation diagonalizes under the similarity transform, $A(\mathbf{n})=S^{-1}(\mathbf{n}) \Lambda(\mathbf{n}) S(\mathbf{n})$, where the diagonal eigenvalue matrix, $\Lambda(\mathbf{n})$, has the entries $\Lambda(\mathbf{n})=|\mathbf{n}| \operatorname{diag}\left(-n^{-1}, 0, n^{-1}\right)$ corresponding to the characteristic velocities of the waves counterpropagating, nonpropagating, and copropagating along the normal vector $\mathbf{n}$ with the local speed of light. Here $|\mathbf{n}|$ represents the length of the vector $\mathbf{n}$ such that $\mathbf{n}=|\mathbf{n}|\left(\hat{n}_{z}, \hat{n}_{x}\right)$. form

The diagonalizing matrices, $S(\mathbf{n})$ and $S^{-1}(\mathbf{n})$, take the

$$
\begin{gathered}
S(\mathbf{n})=\left(\begin{array}{ccc}
-\hat{n}_{x} & \hat{n}_{z} & -\hat{n}_{x} \\
\hat{n}_{z} & \hat{n}_{x} & \hat{n}_{z} \\
n^{-1} & 0 & -n^{-1}
\end{array}\right), \\
S^{-1}(\mathbf{n})=\frac{1}{2}\left(\begin{array}{ccc}
-\hat{n}_{x} & \hat{n}_{z} & n \\
2 \hat{n}_{z} & 2 \hat{n}_{x} & 0 \\
-\hat{n}_{x} & \hat{n}_{z} & -n
\end{array}\right),
\end{gathered}
$$

from which we obtain the characteristic variables

$$
\mathbf{R}=S^{-1}(\mathbf{n}) \mathbf{q}=\left(\begin{array}{l}
R_{1} \\
R_{2} \\
R_{3}
\end{array}\right)=\frac{1}{2}\left(\begin{array}{c}
-\hat{n}_{x} H_{z}+\hat{n}_{z} H_{x}+n E_{y} \\
2 \hat{n}_{z} H_{z}+2 \hat{n}_{x} H_{x} \\
-\hat{n}_{x} H_{z}+\hat{n}_{z} H_{x}-n E_{y}
\end{array}\right) .
$$

The characteristic variables, $\mathbf{R}$, are convected along the normal, $\hat{\mathbf{n}}$, with a speed given by the diagonal elements of $\Lambda(\mathbf{n})$. Hence, once the outward normal vector at the enclosing boundary of the subdomain is known, as it is once the map, $\Psi$, is constructed, those characteristics that are leaving and those that are entering the subdomain (and which thus need specification) may be uniquely determined. Indeed, we observe from the eigenvalues of $A$ that while $R_{3}$ is always leaving the domain and therefore needs no boundary conditions, $R_{1}$ is always entering the computational domain and requires specification to ensure well-posedness. Thus $R_{3}$, leaving a domain, supplies the sought-after boundary conditions for $R_{1}$ in the neighboring domain and conversely for $R_{1}$ in the first domain. For the nonpropagating $R_{2}$ we simply use the average across the interface. Once the characteristic variables have been adjusted, the physical fields are simply recovered through the relation $S(\mathbf{n}) \mathbf{R}=\mathbf{q}$. This procedure is applied along all the interface points, including the vertices, where it is done dimension by dimension, to arrive at the global solution at each time step. As we shall see shortly, this procedure of patching hyperbolic systems is stable as well as accurate. Moreover, in a parallel setting the communication between subdomains grows with the surface of the geometric building block rather than with the volume.

\section{Open Boundary Conditions}

To simulate open boundary conditions on the edges of the global computational domain, we employ a so-called matched layer (ML) technique that was introduced in Ref. 19.

In the ML approach an absorbing term is added to the hyperbolic system, Eq. (14), so that this takes the form

$$
\frac{\partial \mathbf{q}}{\partial t}+A(\nabla \xi) \frac{\partial \mathbf{q}}{\partial \xi}+A(\nabla \eta) \frac{\partial \mathbf{q}}{\partial \eta}-f(z, x) \mathbf{q}=0,
$$

where $f(z, x) \geqslant 0$ in the outer domains and $f(z, x)=0$ at the interface between the outer and the inner domains, as well as in all the inner domains. $f$ is chosen to be some smooth function, which starts deviating from 0 at some distance from the boundary to the inner domain and then grows toward the outer boundary. We use this absorption in combination with a cubic mapping of the grid in the outer domains. This cubic mapping stretches the grid so that it becomes coarse toward the outer boundary. This means that waves propagating in this area will appear as high-frequency waves, and, with a low-pass filtering, the reflections in the ML layer can be effectively filtered out.

\section{E. Near- and Far-Field Calculations}

We wish to calculate the optical field at any distance away from the DOE. Rather than extending the computational domain to cover the whole region of interest, we have chosen to employ the surface equivalence theorem. ${ }^{20}$ This widely used theorem states that equivalent current sources can be calculated on an imaginary surface surrounding a source distribution and that, from these equivalent sources, the field outside the imaginary surface can be calculated.

We employ this method for phasor quantities, so we have to compute the steady-state solution and from that calculate the phasors of the field components. The field may now be found anywhere outside the imaginary surface by integration of the equivalent sources weighted by the two-dimensional free-space Green's function. The integration can be performed with spectral accuracy, since integration weights for the Gauss-Lobatto grid exist. Using this integration technique not only is faster than using the spectral method to propagate the solution but also saves a great deal of computation time and memory, since we do not have to calculate the field components in all the intermediate regions in determining, for example, the focal distance of a FGC.

In the far field the integration simplifies, since the integrals, which in the general case depend on both source and observation point locations, can be made to depend only on the source point location. For a detailed description of this method we refer to the reader to Ref. 13.

One problem arises when implementing the surface equivalence theorem in our context: A prerequisite for 
the method is that the virtual surface is closed. However, for our problem, the thin-film waveguide must be of infinite extent to avoid reflections, and hence we can realize the surface only as a plane (or, in two dimensions, as an open contour) above the closed structure. As we shall demonstrate, this does not lead to a significant loss of accuracy.

\section{NUMERICAL RESULTS}

As a first test case, we employ our pseudospectral method with respect to a simple four-layer thin-film waveguide for which an analytic solution exists. ${ }^{21}$ The waveguide structure consists of a core layer of refractive index $n$ $=1.45$ and thickness $d_{1}=0.8 \lambda$ sandwiched between two cladding layers of refractive index $n=1.4$. The top cladding layer has a finite thickness of $d_{2}=\lambda$, and above this layer is air with $n=1$. The pseudospectral grid for this structure is shown in Fig. 3.

We initialize the field components in the waveguide structure with the analytic solution and let the field march on in time. At $t=10$ we compare the numerical and the analytic solutions; the results are shown in Table 1 in terms of the global $L_{\infty}$ error of all three field components. The solutions are, naturally, compared only in the interior domains and not in the outer domains, where the absorption term has been added to the equations.

From Table 1 it can be seen that there is excellent agreement between our numerical solution and the analytic solution even for as few as 7 points/wavelength. We have also performed a verification of the propagation constant of the propagating field, and we find that the rela-

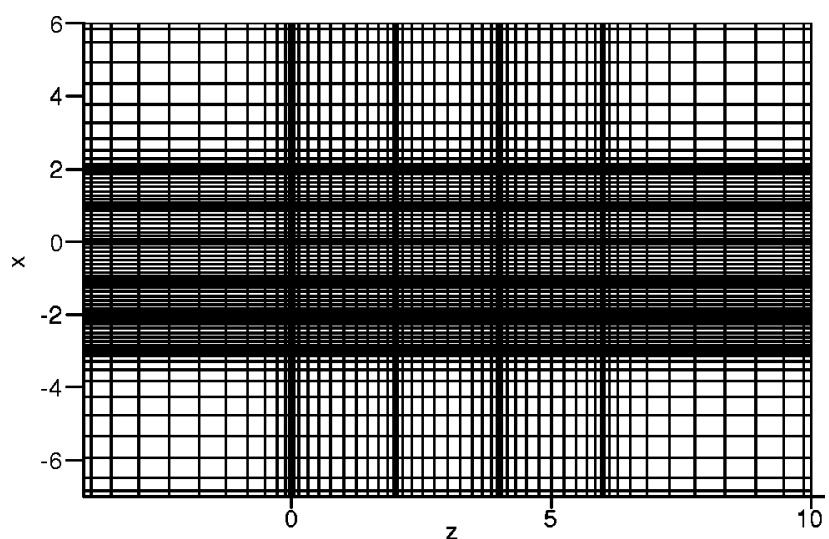

Fig. 3. Pseudospectral grid for analysis of a thin-film optical waveguide. The axis coordinates are normalized as described in Section 2.

Table 1. Global $L_{\infty}$ Error of All Three Field Components for a Thin-Film Waveguide Problem as a Function of the Number of Grid Points per Wavelength

\begin{tabular}{cccccc}
\hline$N$ & $N_{\text {ppw }}$ & $\Delta t$ & $L_{\infty}\left(H_{z}\right)$ & $L_{\infty}\left(H_{x}\right)$ & $L_{\infty}\left(E_{y}\right)$ \\
\hline 12 & 4.2 & $3.6 \times 10^{-2}$ & $4.5 \times 10^{-2}$ & $3.4 \times 10^{-1}$ & $2.4 \times 10^{-1}$ \\
16 & 5.7 & $2.1 \times 10^{-2}$ & $1.0 \times 10^{-3}$ & $8.5 \times 10^{-3}$ & $6.0 \times 10^{-3}$ \\
20 & 7.1 & $1.6 \times 10^{-2}$ & $7.4 \times 10^{-6}$ & $5.5 \times 10^{-5}$ & $3.9 \times 10^{-5}$ \\
24 & 8.6 & $1.2 \times 10^{-2}$ & $1.9 \times 10^{-6}$ & $1.7 \times 10^{-5}$ & $1.2 \times 10^{-5}$ \\
\hline
\end{tabular}

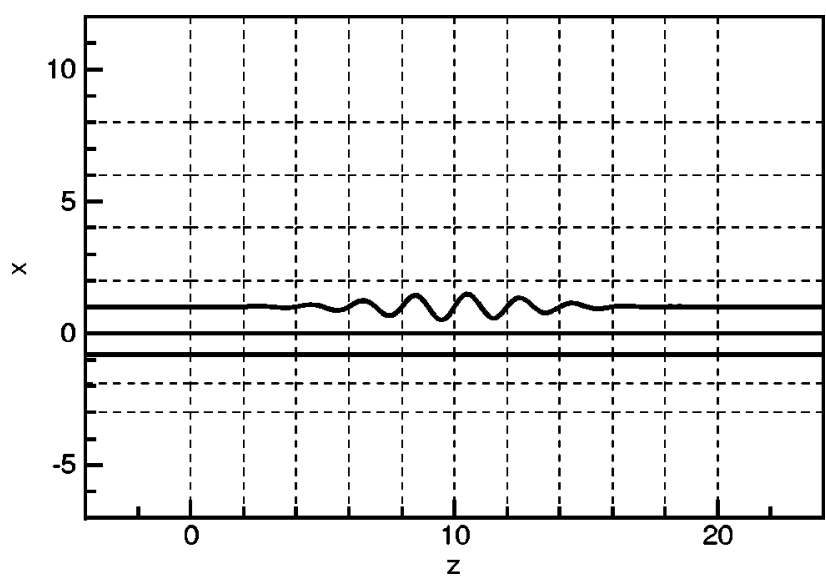

Fig. 4. Grating coupler consisting of a thin-film waveguide with a corrugated top cladding layer. The domain decomposition of the computational domain is indicated.

tive deviation from the semianalytically determined propagation constant lies within $6 \times 10^{-4}$ for a resolution of 7.1 points/free-space wavelength.

We now introduce a continuous surface relief in the top cladding layer with the generic expression for the height perturbation

$$
\begin{aligned}
h(z)= & A \exp \left[-\left(\frac{z-z_{0}}{w}\right)^{2}\right] \\
& \times \cos \left\{2 \pi\left[a_{0}+a_{1}\left(z-z_{0}\right)\right]\left(z-z_{0}\right)\right\},
\end{aligned}
$$

where $A$ is the amplitude, $w$ is the width of the exponentially truncated relief, $z_{0}$ is the center of the relief, $a_{0}$ is the inverse period of the unchirped relief, and $a_{1}$ is a chirp parameter. We choose to investigate an analog relief, since this yields higher diffraction efficiencies. Furthermore, in our department at the Ris $\emptyset$ National Laboratory we have facilities for producing DOE's comprising analog surface reliefs.

The first example employing the surface relief is the grating coupler shown in Fig. 4. The decomposition of the global computational domain into subdomains and the layers of the thin-film waveguide are indicated. The parameters are $A=0.5, w=4, z_{0}=10, a_{0}=0.7$, and $a_{1}=0$. The total length of the structure is $20 \lambda$.

Figure 5 shows a snapshot of the electrical-field component in the structure. One can clearly see the radiation from the surface-relief grating, and one can also get an idea of the -1 st-order diffraction angle.

We now perform a self-consistency verification of our numerical scheme by comparing the direct solution along the line $x=6$ with the solution that one obtains by calculating equivalent current sources on a virtual aperture located at $x=2$ and then integrating this solution to $x$ $=6$, using the method outlined in Section 3. This comparison can be seen in Fig. 6, which shows the amplitude of the complex field phasors of the steady-state solution for all three field components. It can be seen that there is excellent agreement between the two solutions. This agreement demonstrates that the fact that we cannot use a closed surface in the implementation of the surface equivalence theorem does not significantly influence the accuracy of this free-space integration. It can also be 
concluded from the excellent agreement that the reflections from the outer boundaries are efficiently suppressed, since such reflections would give rise to a disagreement between the two solutions. For a detailed analysis of the ML accuracy, we refer the reader to Ref. 14.

To further verify the numerical scheme, we perform a computation of the far-field radiation pattern of the grating coupler. The diffraction orders of such a surfacerelief grating can be found from

$$
n_{\mathrm{eff}}+\frac{m \lambda}{\Lambda}=\sin \theta_{m},
$$

where $m$ is the diffraction order, $\theta_{m}$ is the corresponding radiation angle, and $n_{\text {eff }}$ is the effective refractive index of the unperturbed thin-film waveguide. This effective index is found from the semianalytic solution for the unperturbed waveguide.

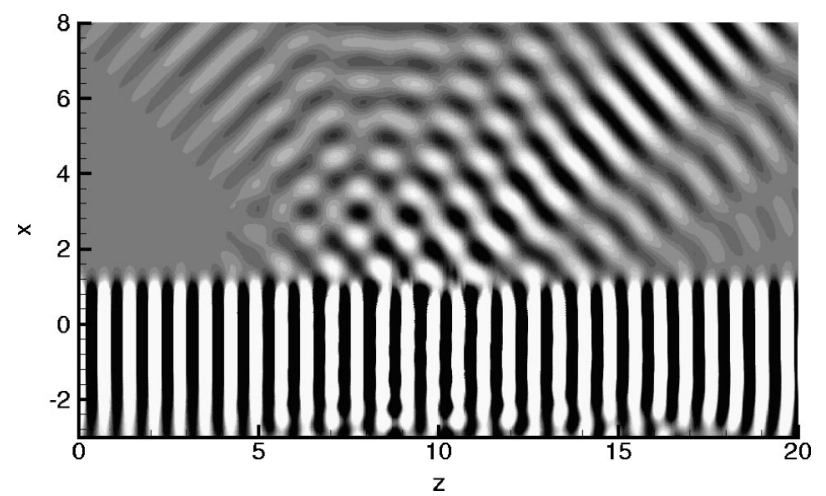

Fig. 5. Contour plot showing a snapshot of the $E_{y}$ field component in a grating coupler. The dark shading corresponds to large negative values of the field, while the light shading corresponds to large positive values.
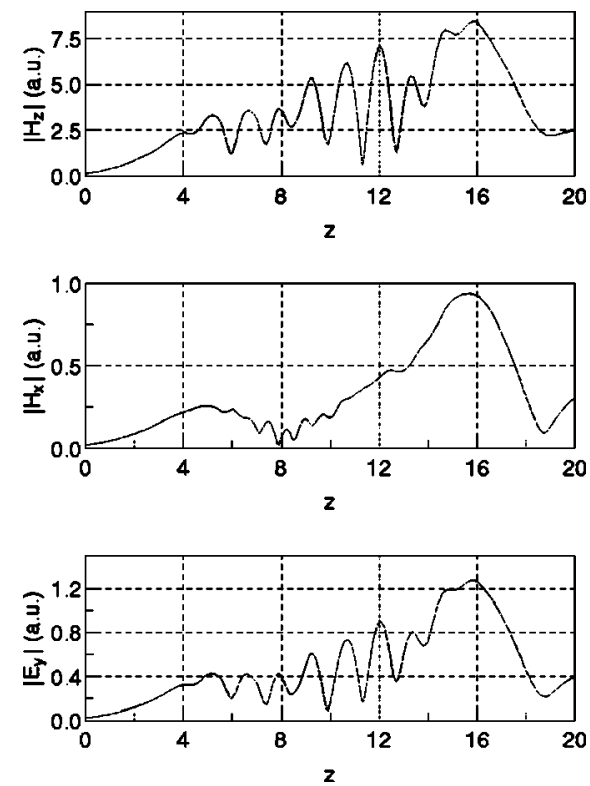

Fig. 6. Comparison of the direct solution and the solution obtained by free-space integration for a grating coupler, for field components (a) $H_{z}$, (b) $H_{x}$, and (c) $E_{y}$. Solid curve, direct solution; dashed curve, solution obtained by free-space integration (solid and dashed curves are nearly indistinguishable).

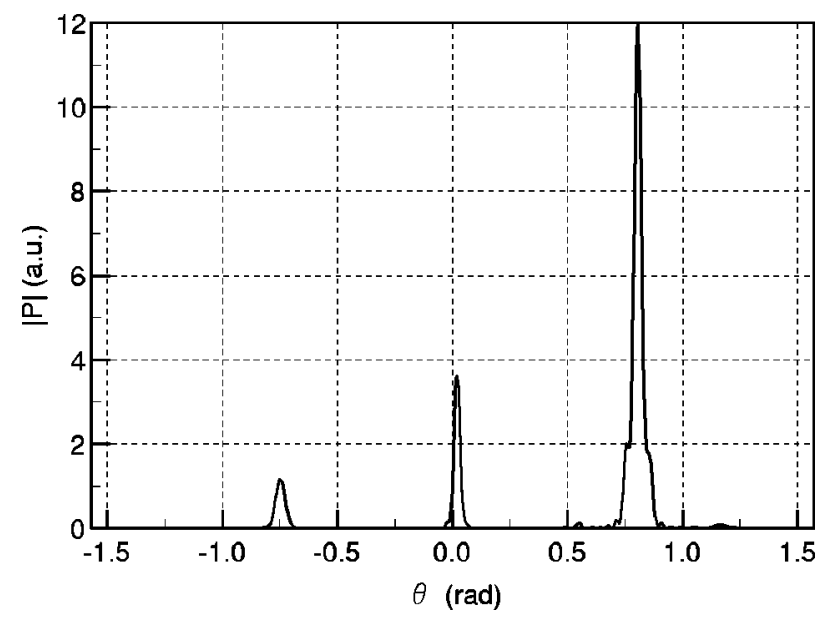

Fig. 7. Far-field radiation pattern from a grating coupler.

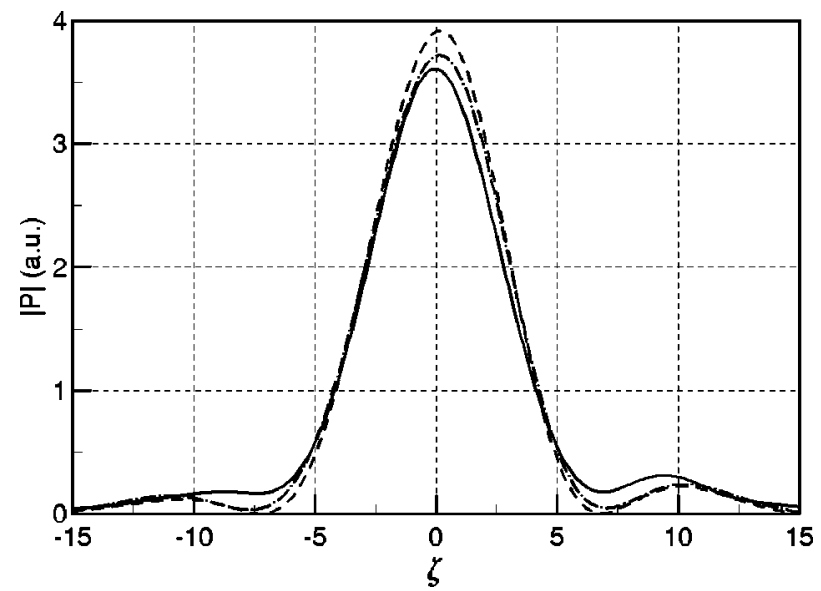

Fig. 8. Line scan of the Poynting vector in the focal plane of the -1st diffraction order for a FGC. Distance from structure:

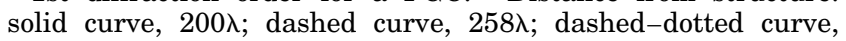
$300 \lambda$.

To make the far-field pattern more distinct, a higher number of oscillations is needed, and hence we perform the far-field calculation for a structure of length $40 \lambda$ with $w=16$.

The far-field radiation pattern, depicted as the normalized amplitude of the Poynting vector as a function of the radiation angle $\theta$, is shown in Fig. 7. If we now compare the diffraction angles corresponding to the -1 st, -2 nd, and -3 rd orders, read from this figure with those found from Eq. (18), we find that the deviations all lie within $5 \times 10^{-4}$.

For a last example we turn to a FGC, where we set the chirp parameter in Eq. (17) to $a_{1}=0.0005$. The length of the FGC is $80 \lambda$, and $w=24$. We find the steady-state solution and use the free-space integration technique described above to determine the focal plane of the output. Figure 8 shows a line scan of the Poynting vector at three different distances from the FGC. The line scan is along a line perpendicular to the unperturbed diffraction angle of the -1st diffraction order with the local length variable $\zeta$. From this figure it can be seen that the output field has a waist at a distance of $258 \lambda$ from the surface relief. This focal distance is significantly lower than the focal distance found by application of simple ray-tracing con- 
siderations, which gives $470 \lambda$. A similar focal shift has previously been observed for low-Fresnel-number diffractive lenses. ${ }^{5}$ The width of the beam in the focal plane is $9.7 \lambda$, which is approximately $50 \%$ larger than the diffraction spot size, found to be $7 \lambda$, based on the $1 / e^{2}$ width of the Gaussian truncation of the surface relief.

\section{CONCLUSIONS}

We have proposed the use of a pseudospectral method for the analysis of diffractive optical elements. This approach is based on a spectral computation of the spatial derivatives in the time-domain Maxwell equation. By use of a curvilinear representation of the equations in a multidomain formulation, the method is applicable for modeling field propagation in a wide range of diffractive optical elements.

For a first test case we demonstrated the ability of our approach to accurately compute the propagation in a thinfilm optical waveguide. For this problem an analytic solution exists, and we find that, with the use of as little as 7 points/free-space wavelength, our method can accurately resolve the solution to this problem.

For a second example we modeled a grating coupler comprising a surface relief for out-of-plane coupling. We demonstrated consistency between our method and a method based on the surface equivalence principle. We further calculated the far-field radiation pattern of the grating coupler and found excellent agreement with the theoretical output angles of three diffraction orders.

For a final example we used that of a focusing grating coupler. We calculated the focal distance and the beamwaist diameter and found that the focal distance calculated by this method is significantly lower than the distance predicted by simple geometrical optics, whereas the beam-waist diameter is $50 \%$ above the diffraction spot size.

\section{ACKNOWLEDGMENTS}

We are grateful to Steen G. Hansson for many fruitful discussions on diffractive optics. The partial support of the work of J. Hesthaven through Defense Advanced Research Project Agency/U.S. Air Force Office of Scientific Research grant F49620-96-1-0426, Department of Energy grant DE-FG02-95ER25239, and National Science Foundation grant ASC-9504002 is gratefully acknowledged.

\section{REFERENCES}

1. M. W. Farn, "Binary gratings with increased efficiency," Appl. Opt. 31, 4453-4458 (1992).

2. T. K. Gaylord and M. G. Moharam, "Analysis and applications of optical diffraction by gratings," Proc. IEEE $\mathbf{7 3}$ 894-937 (1985).

3. K. Hirayama, E. N. Glytsis, and T. K. Gaylord, "Rigorous electromagnetic analysis of diffraction by finite-number-ofperiods gratings," J. Opt. Soc. Am. A 14, 907-917 (1997).

4. M. S. Mirotznik, D. W. Prather, and J. N. Mait, "A hybrid finite element-boundary element method for the analysis of diffractive elements," J. Mod. Opt. 43, 1309-1321 (1996).

5. D. W. Prather, M. S. Mirotznik, and J. N. Mait, "Boundary integral methods applied to the analysis of diffractive optical elements," J. Opt. Soc. Am. A 14, 34-43 (1997).

6. M. Schmitz and O. Bryngdahl, "Rigorous concept for the design of diffractive microlenses with high numerical apertures," J. Opt. Soc. Am. A 14, 901-906 (1997).

7. M. Mirotznik, J. N. Mait, D. W. Prather, and W. A. Beck, "Three-dimensional vector-based analysis of subwavelength diffractive optical elements using the finitedifference time-domain (FDTD) method," in Diffractive Optics and Micro-Optics, Vol. 10 of 1998 OSA Technical Digest Series (Optical Society of America, Washington, D.C., 1998), pp. 91-93.

8. S. Ura, T. Suhara, and H. Nishihara, "Aberration characterizations of a focusing grating coupler in an integratedoptic disk pickup device," Appl. Opt. 26, 4777-4782 (1987).

9. J. J. M. Braat and M. O. E. Laurijs, "Geometrical optics design and aberration analysis of a focusing grating coupler," Opt. Eng. 33, 1037-1043 (1994).

10. P.-P. Borsboom and H. J. Frankena, "Field analysis of twodimensional focusing grating couplers," J. Opt. Soc. Am. A 12, 1142-1146 (1995).

11. R. W. Ziolkowski, "The incorporation of microscopic material models into the FDTD approach for ultrafast optical pulse simulations," IEEE Trans. Antennas Propag. 45, 375-391 (1997).

12. B. Yang, D. Gottlieb, and J. S. Hesthaven, "Spectral simulation of electromagnetic wave scattering," J. Comput. Phys. 134, 216-230 (1997).

13. A. Taflove, Computational Electrodynamics-The FiniteDifference Time-Domain Method (Artech House, Boston, Mass., 1995).

14. J. S. Hesthaven, P. G. Dinesen, and J.-P. Lynov, "Parallel pseudospectral time-domain modeling of diffractive optical elements," submitted to J. Comput. Vision.

15. M. H. Carpenter and C. A. Kennedy, "Fourth order $2 N$-storage Runge-Kutta scheme," Tech. Rep. NASA-TM109112 (NASA, Washington, D.C., 1994).

16. D. Funaro, Polynomial Approximation of Differential Equations, Vol. 8 of Lecture Notes in Physics (Springer-Verlag, Berlin, 1992)

17. W. J. Gordon and C. A. Hall, "Transfinite element methods: blending-function interpolation over arbitrary curved element domains," Numer. Math. 21, 109-129 (1973).

18. J. S. Hesthaven, "A stable penalty method for the compressible Navier-Stokes equations. III. Multi-dimensional domain decomposition schemes," SIAM (Soc. Ind. Appl. Math.) J. Sci. Comput. 20, 62-93 (1999).

19. B. Yang, D. Gottlieb, and J. S. Hesthaven, "On the use of PML ABC's in spectral time-domain simulations of electromagnetic scattering," in Proceedings of the 13th Annual Review of Progress in Applied Computational Electromagnetics (ACES '97), E. C. Michielssen, ed. (Applied Computational Electromagnetics Society, Monterey, Calif., 1997), Vol. 2, pp. 926-933.

20. S. A. Schelknuoff, "Some equivalence theorems of electromagnetics and their application to radiation problems," Bell Syst. Tech. J. 15, 92-112 (1936).

21. S. Ramo, J. R. Whinnery, and T. van Duzer, Fields and Waves in Communications Electronics, 3rd ed. (Wiley, New York, 1993). 